\title{
WEIGHTED EMBEDDING THEOREMS FOR RADIAL BESOV AND TRIEBEL-LIZORKIN SPACES
}

\author{
PABLO L. DE NÁPOLI, IRENE DRELICHMAN, AND NICOLAS SAINTIER
}

\begin{abstract}
We study the continuity and compactness of embeddings for radial Besov and Triebel-Lizorkin spaces with weights in the Muckenhoupt class $A_{\infty}$. The main tool is a discretization in terms of an almost orthogonal wavelet expansion adapted to the radial situation.
\end{abstract}

\section{INTRODUCTION}

Weighted embedding theorems for smooth function spaces have beeen studied by many authors, mainly because they are a fundamental tool in the variational analysis of some nonlinear partial differential equations, for instance of degenerate or singular elliptic equations. It is therefore natural to study embedding results in the framework of Triebel-Lizorkin and Besov spaces, since these include many of the classical functional spaces. In the unweighted case, a fundamental result in this context is the embedding theorem of Jawerth [14] and Franke [10], which generalizes the classical Sobolev embedding theorem.

Weighted Besov and Triebel-Lizorkin spaces have also been studied by many authors under different assumptions on the weights (see e.g. [2, 3, 25]). Embeddings of Besov and Triebel-Lizorkin spaces with Muckenhoupt's $\mathcal{A}_{\infty}$ weights were studied by Haroske and Skrzypczak in [12, 13] and Meyries and Veraar in [20] (see also [19] for an earlier work by the same authors in the case of power weights).

On the other hand, it is well known, since the pioneering works of $\mathrm{Ni}$ [21] and Strauss [29], that many embedding results can be improved when one considers subspaces of radial functions. More precisely, by restricting ourselves to the subspace of radial functions, we can recover, for instance, compactness properties of embeddings that are in general non-compact due to the action of some non-compact group of transformations such as the group of translations in $\mathbb{R}^{n}$ (see, e.g. [18]). Notice that compact embeddings are a fundamental feature for the success of variational methods in PDE. In the case of weighted embedding theorems one can also obtain a wider range of exponents for the admissible power weights in the radial situation (see e.g. [6]).

In the case of unweighted radial subspaces of Besov and Triebel-Lizorkin spaces, Sickel and Skrzypczak [26, 27] and Sickel, Skrzypczak and Vybiral [28] obtained compactness of the related embeddings and an extension of Strauss' radial lemma.

1991 Mathematics Subject Classification. 46E35, 42C40.

Key words and phrases. embedding theorems, radial functions, Muckenhoupt weights, wavelet bases.

Supported by ANPCyT under grant PICT 1675/2010, by CONICET under grant PIP 1420090100230 and by Universidad de Buenos Aires under grant 20020090100067. The authors are members of CONICET, Argentina. 
Quantitative information in terms of entropy numbers for the embeddings was obtained by Kühn, Leopold, Sickel and Skrzypczak in [16]. In these papers, the main tool is an atomic decomposition adapted to the radial situation.

Early results on embeddings for weighted radial Besov and Triebel-Lizorkin spaces can be found in Triebel's book [31, Section 6.5.2], where the weights considered are of the special forms $w_{\alpha}(x)=\left(1+|x|^{2}\right)^{\alpha / 2}$ with $\alpha \in \mathbb{R}$, and $w^{\beta}(x)=e^{|x|^{\beta}}$ with $|x| \geq 1$ and $0<\beta \leq 1$ (see also references therein). However, to the authors knowledge, results on weighted radial Besov and Triebel-Lizorkin spaces for other important classes of weights, such as power weights or, more generally, Muckenhoupt weights, were still missing in the literature. The first two authors recently showed in [7] that the approach used by Meyries and Veraar [19] to obtain embedding theorems with power weights can be improved to obtain a better range of admissible exponents in the radial case. In this work we consider embedding theorems for radial subspaces of Besov and Triebel-Lizorkin spaces with general $\mathcal{A}_{\infty}$ weights. It is important to stress that in the latter case the functions considered are radially symmetric, but the weights can be arbitrary. In the Triebel-Lizorkin case, we follow an argument by Meyries and Veraar [20] to derive the embeddings from the Besov case, but this time restrict ourselves not only to radially symmetric functions but also to radially symmetric $\mathcal{A}_{\infty}$ weights (see the discussion in Section $5)$. In both cases we obtain sufficient conditions for the continuity and compactness of the embeddings that improve with respect to the non-radial case.

For our proof, instead of using the atomic decomposition for radial subspaces of Sickel and Skrzypczak [26], we shall follow closely the approach used by Haroske and Skrzypczak $[12,13]$ in the non-radial case, which is based on a discretization in terms of wavelet bases. To this end, we need a wavelet decomposition adapted to the radial situation, which we obtain by adapting arguments used by Epperson and Frazier [9] in the unweighted radial case. We remark that this is not a wavelet decomposition in the traditional way, since the wavelets are localized near certain annuli instead of cubes. Hence, they have the advantage of being better adapted to the radial situation but have no translation structure and, more importantly, since they are not actual bases but rather frames, they do not characterize the (weighted) Besov and Triebel-Lizorkin spaces. In other words, they are useful to obtain sufficient conditions for the continuity and compactness of the embeddings, but cannot be used to prove sharpness of the conditions obtained. Unfortunately, as far we know, there are no known orthogonal wavelet decompositions for radial functions except in dimension three (see, e.g. $[24,4]$ ).

The rest of the paper is as follows. In Section 2 we recall some definitions and known properties of Besov and Triebel-Lizorkin spaces. Section 3 in devoted to the construction of the wavelet bases and the representation of the weighted radial Besov and Triebel-Lizorkin spaces in terms of sequence spaces (Theorems 3.1 and 3.2). In Section 4 we prove our main theorem (Theorem 4.1) on sufficient conditions for the continuity and compactness of the embeddings for weighted radial Besov spaces and use it to analyze some important special examples. Finally, in Section 5 we obtain sufficient conditions for the continuity and compactness of the embeddings for Triebel-Lizorkin spaces with radial $\mathcal{A}_{\infty}$ weights (Theorem 5.1) and an example in this case. 


\section{Weighted Besov AND TRIEBEL-Lizorkin SPACES}

First we recall some necessary definitions. For classical references on Besov and Triebel-Lizorkin spaces see $[22,30]$. For weighted versions see [3, 25].

Definition 2.1 (Construction of the Littlewood-Paley partition). Let $\varphi \in \mathscr{S}\left(\mathbb{R}^{n}\right)$ be such that

$$
\begin{aligned}
& (2.1) \quad 0 \leq \widehat{\varphi}(\xi) \leq 1, \quad \xi \in \mathbb{R}^{n}, \quad \widehat{\varphi}(\xi)=1 \quad \text { if }|\xi| \leq 1, \quad \widehat{\varphi}(\xi)=0 \quad \text { if }|\xi| \geq \frac{3}{2} . \\
& \text { Let } \widehat{\varphi}_{0}=\widehat{\varphi}, \widehat{\varphi}_{1}(\xi)=\widehat{\varphi}(\xi / 2)-\widehat{\varphi}(\xi), \text { and } \\
& \widehat{\varphi}_{\mu}(\xi)=\widehat{\varphi}_{1}\left(2^{-\mu+1} \xi\right)=\widehat{\varphi}\left(2^{-\mu} \xi\right)-\widehat{\varphi}\left(2^{-\mu+1} \xi\right), \quad \xi \in \mathbb{R}^{n}, \quad \mu \geq 1 .
\end{aligned}
$$

Then

$0 \leq \widehat{\varphi}_{\mu}(\xi) \leq 1, \quad \widehat{\varphi}_{\mu}(\xi)=1 \quad$ if $\frac{3}{2} 2^{\mu-1} \leq|\xi| \leq 2^{\mu}, \quad$ supp $\widehat{\varphi}_{\mu} \subset\left\{2^{\mu-1} \leq|\xi| \leq \frac{3}{2} 2^{\mu}\right\}$.

Let $\Phi$ be the set of all sequences $\left(\varphi_{\mu}\right)_{\mu \geq 0}$ constructed in the above way from a function $\varphi$ that satisfies (2.1).

For $\varphi$ as in the definition and $f \in \mathscr{S}^{\prime}\left(\mathbb{R}^{n}\right)$ one sets

$$
S_{\mu} f:=\varphi_{\mu} * f=\mathscr{F}^{-1}\left[\widehat{\varphi}_{\mu} \widehat{f}\right],
$$

which belongs to $C^{\infty}\left(\mathbb{R}^{n}\right) \cap \mathscr{S}^{\prime}\left(\mathbb{R}^{n}\right)$. Since $\sum_{\mu \geq 0} \widehat{\varphi}_{\mu}(\xi)=1$ for all $\xi \in \mathbb{R}^{n}$, we have $\sum_{\mu \geq 0} S_{\mu} f=f$ in the sense of distributions.

Given a weight $w$, that is a non-negative locally integrable function on $\mathbb{R}^{n}$, and a real number $p \in[1,+\infty]$, we denote by $L^{p}\left(\mathbb{R}^{n}, w\right)$ the weighted Lebesgue space defined as the space of those measurable functions $f: \mathbb{R}^{n} \rightarrow \mathbb{R}$ such that

$$
\|f\|_{L^{p}\left(\mathbb{R}^{n}, w\right)}^{p}:=\int_{\mathbb{R}^{n}}|f|^{p} w(x) d x<\infty
$$

if $1 \leq p<+\infty$, and $\|f\|_{L^{\infty}\left(\mathbb{R}^{n}, w\right)}=\|f\|_{L^{\infty}\left(\mathbb{R}^{n}\right)}$.

Let us recall that, for $1<p<+\infty$, the Muckenhoupt class $\mathcal{A}_{p}$ is the class of weights $w$ for which the maximal Hardy-Littlewood operator is bounded from $L^{p}\left(\mathbb{R}^{n}, w\right)$ to itself, and that it can be characterized by the condition

$$
\left(\frac{1}{|B|} \int_{B} w\right)\left(\frac{1}{|B|} \int_{B} w^{1-p^{\prime}}\right)^{p-1} \leq C
$$

for all balls $B \subseteq \mathbb{R}^{n}$, where the constant $C$ depends on $w$ but is independent of $B$. On the other hand, we say that $w \in \mathcal{A}_{1}$ if $M w(x) \leq C w(x)$ a.e., and we set $\mathcal{A}_{\infty}=\bigcup_{p \geq 1} \mathcal{A}_{p}$. We refer to [11] for a detailed account of these weights.

Given real numbers $p, q \in[1, \infty], s \in \mathbb{R}$ and a weight $w \in \mathcal{A}_{\infty}$, we can define following [2] the weighted Besov and Triebel-Lizorkin spaces $B_{p, q}^{s}\left(\mathbb{R}^{n}, w\right)$ and $F_{p, q}^{s}\left(\mathbb{R}^{n}, w\right)$ in the following way

Definition 2.2. The (inhomogeneous) Besov space $B_{p, q}^{s}\left(\mathbb{R}^{n}, w\right)$ is defined as the space of all $f \in \mathscr{S}^{\prime}\left(\mathbb{R}^{n}\right)$ for which

$$
\|f\|_{B_{p, q}^{s}\left(\mathbb{R}^{n}, w\right)}:=\left(\sum_{\mu \geq 0} 2^{q \mu s}\left\|S_{\mu} f\right\|_{L^{p}\left(\mathbb{R}^{n}, w\right)}^{q}\right)^{1 / q}<\infty .
$$

with the usual modifications for $q=\infty$. 
Definition 2.3. Assume that $p<\infty$. The (inhomogeneous) Triebel-Lizorkin space $F_{p, q}^{s}\left(\mathbb{R}^{n}, w\right)$ is defined as the space of all $f \in \mathscr{S}^{\prime}\left(\mathbb{R}^{n}\right)$ for which

$$
\|f\|_{F_{p, q}^{s}\left(\mathbb{R}^{n}, w\right)}:=\left\|\left(\sum_{\mu \geq 0} 2^{q \mu s}\left|S_{\mu} f\right|^{q}\right)^{1 / q}\right\|_{L^{p}\left(\mathbb{R}^{n}, w\right)}<\infty .
$$

with the usual modifications for $q=\infty$.

Remark 2.1. (1) It can be proved that these definitions do not depend on the choice of the particular $\varphi$ in (2.1), see e.g. [3].

(2) The corresponding homogeneous spaces denoted by $\dot{B}_{p, q}^{s}\left(\mathbb{R}^{n}, w\right)$ and $\dot{F}_{p, q}^{s}\left(\mathbb{R}^{n}, w\right)$ are defined in a similar way with the sum running over $\mathbb{Z}$ with appropriate modifications of the partition of unity. Observe that $\|f\|_{B_{p, q}^{s}\left(\mathbb{R}^{n}, w\right)}=0$ if and only if supp $\hat{f}=\{0\}$, i.e., $f$ is a polynomial. For this reason it is usual to consider instead the quotient spaces $\dot{B}_{p, q}^{s}\left(\mathbb{R}^{n}, w\right) / \mathcal{P}$ and $\dot{F}_{p, q}^{s}\left(\mathbb{R}^{n}, w\right) / \mathcal{P}$ where $\mathcal{P}$ is the space of polynomials.

(3) If $w \equiv 1$, we write $B_{p, q}^{s}\left(\mathbb{R}^{n}\right)$ instead of $B_{p, q}^{s}\left(\mathbb{R}^{n}, w\right)$ and $F_{p, q}^{s}\left(\mathbb{R}^{n}\right)$ instead of $F_{p, q}^{s}\left(\mathbb{R}^{n}, w\right)$.

The group $O(n)$ of $\mathbb{R}^{n}$ acts on $\mathscr{S}\left(\mathbb{R}^{n}\right)$ by $(\sigma, \phi) \in O(n) \times \mathscr{S}\left(\mathbb{R}^{n}\right) \rightarrow \sigma \phi \in \mathscr{S}\left(\mathbb{R}^{n}\right)$ with $\sigma \phi(x):=\phi\left(\sigma^{-1} x\right)$. Then, for any $f, \phi \in \mathscr{S}\left(\mathbb{R}^{n}\right)$ and $\sigma \in O(n)$ there holds that $(\sigma . f, \phi)_{L^{2}}=\left(f, \sigma^{-1} \phi\right)_{L^{2}}$. We thus define the action of $O(n)$ on $\mathscr{S}^{\prime}\left(\mathbb{R}^{n}\right)$ by $(\sigma, f) \in O(n) \times \mathscr{S}^{\prime}\left(\mathbb{R}^{n}\right) \rightarrow \sigma . f \in \mathscr{S}^{\prime}\left(\mathbb{R}^{n}\right)$ with

$$
(\sigma . f, \phi):=\left(f, \sigma^{-1} \phi\right) \quad \text { for any } \phi \in \mathscr{S}\left(\mathbb{R}^{n}\right) .
$$

This motivates our next definition:

Definition 2.4. We say that a tempered distribution $f \in \mathscr{S}^{\prime}\left(\mathbb{R}^{n}\right)$ is radial if $\sigma . f=$ $f$ for any $\sigma \in O(n)$ where $\sigma . f$ is defined by (2.2).

The Besov and Triebel-Lizorkin spaces of radial distributions will be denoted by $R B_{p, q}^{s}\left(\mathbb{R}^{n}, w\right)$ and $R F_{p, q}^{s}\left(\mathbb{R}^{n}, w\right)$, respectively. The following embeddings between these spaces are elementary and follow from the corresponding non-radial situation (see [2, Theorem 2.6]).

Theorem 2.1. Let $w \in A_{\infty}$. Then

(1) For all $1 \leq q_{1} \leq q_{2} \leq \infty$ and $s \in \mathbb{R}$ one has

$$
\begin{array}{ll}
R B_{p, q_{1}}^{s}\left(\mathbb{R}^{n}, w\right) \hookrightarrow R B_{p, q_{2}}^{s}\left(\mathbb{R}^{n}, w\right), & p \in[1, \infty] ; \\
R F_{p, q_{1}}^{s}\left(\mathbb{R}^{n}, w\right) \hookrightarrow R F_{p, q_{2}}^{s}\left(\mathbb{R}^{n}, w\right), & p \in[1, \infty] .
\end{array}
$$

(2) For all $q_{1}, q_{2} \in[1, \infty], s \in \mathbb{R}$ and $\varepsilon>0$ one has

$$
\begin{aligned}
& R B_{p, q_{1}}^{s+\varepsilon}\left(\mathbb{R}^{n}, w\right) \hookrightarrow R B_{p, q_{2}}^{s}\left(\mathbb{R}^{n}, w\right), \quad p \in[1, \infty] ; \\
& R F_{p, q_{1}}^{s+\varepsilon}\left(\mathbb{R}^{n}, w\right) \hookrightarrow R F_{p, q_{2}}^{s}\left(\mathbb{R}^{n}, w\right), \quad p \in[1, \infty] .
\end{aligned}
$$

(3) For all $q \in[1, \infty], s \in \mathbb{R}$ and $p \in[1, \infty)$ one has

$$
R B_{p, \min \{p, q\}}^{s}\left(\mathbb{R}^{n}, w\right) \hookrightarrow R F_{p, q}^{s}\left(\mathbb{R}^{n}, w\right) \hookrightarrow R B_{p, \max \{p, q\}}^{s}\left(\mathbb{R}^{n}, w\right) .
$$


We now state a weighted version due to [2] of the continuity of Peetre maximal function originally defined in [23].

Let $a>0$ and $\left\{\phi_{\mu}\right\}_{\mu \geq 0}$ be a sequence of functions in $\mathscr{S}\left(\mathbb{R}^{n}\right)$ such that

$$
\operatorname{supp} \widehat{\phi_{\mu}} \subset\left\{2^{\mu-a} \leq|\xi| \leq 2^{\mu+a}\right\},
$$

and

$$
\left|D^{\alpha} \widehat{\phi_{\mu}}(\xi)\right| \leq C_{n} 2^{-\mu|\alpha|} \quad \text { for all } \mu \geq 0, \alpha \in \mathbb{N}^{d}, \xi \in \mathbb{R}^{n} .
$$

This holds e.g. if $\widehat{\phi_{\mu}}(\xi)=\widehat{\phi}_{1}\left(2^{-\mu} \xi\right)$. For a given $\lambda>0$ the Peetre maximal functions of $f \in \mathscr{S}^{\prime}\left(\mathbb{R}^{n}\right)$ are

$$
\phi_{\mu, \lambda}^{*} f(x)=\phi_{\mu}^{*} f(x)=\sup _{y \in \mathbb{R}^{n}} \frac{\left|\phi_{\mu} * f(x-y)\right|}{\left(1+2^{\mu}|y|\right)^{\lambda}}, \quad x \in \mathbb{R}^{n}, \mu \geq 0 .
$$

Theorem 2.2. [2, Section 5] Let $r_{0}=\inf \left\{r: w \in A_{r}\right\}$.

i) If $\lambda>\max \left\{\frac{n r_{0}}{p}, \frac{n}{q}\right\}$ then

$$
\left\|\left(\sum_{\mu \geq 0}\left[2^{\mu s} \phi_{\mu}^{*} f(x)\right]^{q}\right)^{\frac{1}{q}}\right\|_{L_{p}\left(\mathbb{R}^{n}, w\right)} \leq C\|f\|_{F_{p, q}^{s}\left(\mathbb{R}^{n}, w\right)} \quad \text { for all } f \in \mathscr{S}^{\prime}\left(\mathbb{R}^{n}\right) .
$$

ii) If $\lambda>\frac{n r_{0}}{p}$ then

$$
\left(\sum_{\mu \geq 0}\left[2^{\mu s}\left\|\phi_{\mu}^{*} f\right\|_{L_{p}\left(\mathbb{R}^{n}, w\right)}\right]^{q}\right)^{\frac{1}{q}} \leq C\|f\|_{B_{p, q}^{s}\left(\mathbb{R}^{n}, w\right)} \text { for all } f \in \mathscr{S}^{\prime}\left(\mathbb{R}^{n}\right) .
$$

\section{Construction of Radial Wavelets for weighted Besov and} TRIEBEL-LIZORKIN SPACES

In this section we develop a suitable wavelet decomposition adapted to the weighted radial situation. Our starting point is the construction of radial wavelets of Epperson and Frazier [9].

Let $\Phi, \Psi, \varphi, \psi \in \mathscr{S}\left(\mathbb{R}^{n}\right)$ be radial functions such that

$$
\begin{aligned}
\operatorname{supp} \hat{\Phi}, \operatorname{supp} \hat{\Psi} \subset\{|\xi| \leq 1\}, & & |\hat{\Phi}(\xi)|,|\hat{\Psi}(\xi)| \geq c>0 \text { if }|\xi| \leq 5 / 6, \\
\operatorname{supp} \hat{\varphi}, \widehat{\psi} \subset\{1 / 4<|\xi|<1\}, & & |\widehat{\varphi}|,|\widehat{\psi}| \geq c>0 \text { if } 3 / 10 \leq|\xi| \leq 5 / 6,
\end{aligned}
$$

and

$$
\overline{\hat{\Phi}}(\xi) \hat{\Psi}(\xi)+\sum_{\mu \geq 1} \overline{\varphi_{\mu}}(\xi) \widehat{\psi_{\mu}}(\xi)=1 \quad \text { for } \xi \neq 0 .
$$

where $\varphi_{\mu}(x)=2^{\mu n} \varphi\left(2^{\mu} x\right)$ and $\psi_{\mu}(x)=2^{\mu n} \psi\left(2^{\mu} x\right)$. We then define a family of functions $\left(\varphi_{\mu k}\right)_{\mu \geq 0, k \geq 1}$ by

$$
\varphi_{\mu k}= \begin{cases}\left(\frac{2^{(\mu(n-2)+1)}}{j_{\nu, k}^{n} J_{\nu+1}^{2}\left(j_{\nu, k}\right) \omega_{n-1}}\right)^{1 / 2} \varphi_{\mu} * d \sigma_{2^{-\mu} j_{\nu, k}} & \text { for } \mu \geq 1, \\ \left(\frac{2}{j_{\nu, k}^{n} J_{\nu+1}^{2}\left(j_{\nu, k}\right) \omega_{n-1}}\right)^{1 / 2} \Phi * d \sigma_{2^{-\mu} j_{\nu, k}} & \text { for } \mu=0\end{cases}
$$

where $d \sigma_{t}$ denotes the (unnormalized) surface Lebesgue measure on the sphere of radius $t$ in $\mathbb{R}^{n}, \omega_{n-1}$ the surface of the unit sphere, and

$$
0<j_{\nu, 1}<j_{\nu, 2}<\ldots<j_{\nu, k}<\ldots
$$


denote the positive zeros of the Bessel function $J_{\nu}$ with $\nu=(n-2) / 2$. We define in a similiar way the functions $\left(\varphi_{\mu k}\right)_{\mu \geq 0, k \geq 1}$. Then the Epperson-Frazier wavelet expansion for a radial distribution $f \in \mathscr{S}^{\prime}\left(\mathbb{R}^{n}\right)$ is given by

$$
f=\sum_{\mu \geq 0} \sum_{k \geq 1}\left\langle f, \varphi_{\mu, k}\right\rangle \psi_{\mu, k} .
$$

Epperson and Frazier were able to characterize the membership of $f$ to (unweighted) Besov or Triebel-Lizorkin spaces in terms of the wavelet coefficients $\left\langle f, \varphi_{\mu, k}\right\rangle$. Our purpose in this section is to show that analogous results hold for the weighted version of these spaces when the weight belongs to the $\mathcal{A}_{\infty}$ class.

We consider the annuli $A_{\mu, k}, \mu \geq 0, k \geq 1$, defined by

$$
A_{\mu, k}=\left\{x \in \mathbb{R}^{n}, 2^{-\mu} j_{\nu, k-1} \leq|x| \leq 2^{-\mu} j_{\nu, k}\right\} \quad \text { with } j_{\nu, 0}=0,
$$

and denote by $\chi_{\mu, k}:=\left|A_{\mu, k}\right|^{-1 / 2} \chi_{A_{\mu, k}}$ its $L^{2}$-normalized characteristic function. Given real numbers $p, q \in[1, \infty], s \in \mathbb{R}$ and a weight $w \in \mathcal{A}_{\infty}$ we let $b_{p, q}^{s}(w)$ and $f_{p, q}^{s}(w)$ be the spaces of sequences of complex numbers $\lambda:=\left(\lambda_{\mu, k}\right)_{\mu, k}$ such that

$$
\|\lambda\|_{b_{p, q}^{s}(w)}:=\left(\sum_{\mu \geq 0}\left\|\sum_{k \geq 1} 2^{\mu s}\left|\lambda_{\mu, k}\right| \chi_{\mu, k}\right\|_{L_{p}\left(\mathbb{R}^{n}, w\right)}^{q}\right)^{\frac{1}{q}}<\infty,
$$

and

$$
\|\lambda\|_{f_{p, q}^{s}(w)}:=\left\|\left(\sum_{\mu \geq 0} \sum_{k \geq 1}\left[2^{\mu s}\left|\lambda_{\mu, k}\right| \chi_{\mu, k}\right]^{q}\right)^{\frac{1}{q}}\right\|_{L^{p}\left(\mathbb{R}^{n}, w\right)}<\infty
$$

respectively, with the usual modifications if $q=\infty$.

Our first result is the following:

Theorem 3.1. Let $p, q \in[1, \infty]$ and $w \in \mathcal{A}_{\infty}$. Then, the operators

$$
S: f \in R F_{p, q}^{s}\left(\mathbb{R}^{n}, w\right) \rightarrow\left(\left\langle f, \varphi_{\mu, k}\right\rangle\right)_{\mu, k} \in f_{p, q}^{s}(w)
$$

and

$$
T: \lambda \in f_{p, q}^{s}(w) \rightarrow \sum_{\mu \geq 0} \sum_{k \geq 1} \lambda_{\mu, k} \psi_{\mu, k} \in R F_{p, q}^{s}\left(\mathbb{R}^{n}, w\right)
$$

are bounded, and the composition $T \circ S$ is the identity on $R F_{p, q}^{s}\left(\mathbb{R}^{n}, w\right)$. In particular, $\|f\|_{R F_{p, q}^{s}(w)} \simeq\|S(f)\|_{f_{p, q}^{s}(w)}$.

Remark 3.1. The same type of result holds for homogeneous spaces with the usual modification, namely, by summing over $\mu \in \mathbb{Z}$ and suppressing $\Phi$ and $\Psi$.

Proof. The case $w \equiv 1$ corresponds to [9, Theorem 2.1 and 2.2]. Since the proof in the general case is a modification of those results, we sketch it indicating where changes are needed. These mainly concern the continuity of the Peetre maximal function and of the Hardy-Littlewood maximal function for sequences of functions.

Concerning the continuity of $S$ we have as in the proof of [9, Theorem 2.1] that for any $\mu \geq 0$ and $\lambda>0$,

$$
\sum_{k \geq 1}\left(2^{\mu s}\left|\left\langle f, \varphi_{\mu, k}\right\rangle\right| \chi_{\mu, k}(x)\right)^{q} \leq C_{\lambda} 2^{\mu s q}\left|\varphi_{\mu}^{*} f(x)\right|^{q} \text { a.e. }
$$


where $\varphi_{\mu}^{*}$ is the Peetre maximal function as defined in (2.3) for $\lambda>0$. According to Theorem 2.2 we obtain, taking $\lambda$ big enough, that

$$
\|S(f)\|_{f_{p, q}^{s}(w)} \leq C\left\|\left(\sum_{\mu \geq 0} 2^{\mu s q}\left|\varphi_{\mu}^{*} f(x)\right|^{q}\right)^{\frac{1}{q}}\right\|_{L_{p}\left(\mathbb{R}^{n}, w\right)} \leq C\|f\|_{R F_{p, q}^{s}(w)} .
$$

Concerning the continuity of $T$, fix $\lambda \in f_{p, q}^{s}(w)$ and let $f=\sum_{\mu \geq 0} \sum_{k \geq 1} \lambda_{\mu, k} \psi_{\mu, k}$. Then for any $\eta \in(0,1]$ such that $p / \eta, q / \eta>1$ we have as in [9] that

$$
\begin{aligned}
\|f\|_{R F_{p, q}^{s}(w)} & =\left\|\left(\sum_{\mu \geq 0}\left(2^{\mu s}\left|\varphi_{\mu} * f\right|\right)^{q}\right)^{\frac{1}{q}}\right\|_{L^{p}\left(\mathbb{R}^{n}, w\right)} \\
& \leq C\left\|\left(\sum_{\mu \geq 0}\left(M\left(\sum_{k \geq 1}\left(2^{\mu s}\left|\lambda_{\mu, k}\right| \chi_{\mu, k}\right)^{\eta}\right)\right)^{\frac{q}{\eta}}\right)^{\frac{\eta}{q}}\right\|_{L^{\frac{p}{\eta}}\left(\mathbb{R}^{n}, w\right)}^{\frac{1}{\eta}},
\end{aligned}
$$

where $M$ is the Hardy-Littlewood maximal function. According to [1, Theorem 3.1] or [15, Theorem 1], the vector-valued maximal function between weighted spaces

$$
M:\left(f_{\mu}\right)_{\mu} \in L^{\alpha}\left(\ell_{\beta}, w\right) \rightarrow\left(M f_{\mu}\right)_{\mu} \in L^{\alpha}\left(\ell_{\beta}, w\right)
$$

is continuous when the weight $w$ belongs to the $\mathcal{A}_{\alpha}$ class with $1<\alpha, \beta<\infty$. Here $L^{\alpha}\left(\ell^{\beta}\right)$ denotes the space of sequences of locally integrable functions $\left(f_{\mu}\right)_{\mu}$ such that

$$
\left\|\left(f_{\mu}\right)_{\mu}\right\|_{L^{\alpha}\left(\ell_{\beta}, w\right)}^{\alpha}:=\int_{\mathbb{R}^{n}}\left(\sum_{\mu}\left|f_{\mu}\right|^{\beta}\right)^{\frac{\alpha}{\beta}} w d x<\infty .
$$

Since $w \in \mathcal{A}_{p}$, taking $\eta$ small enough to have $p / \eta>r_{0}:=\inf \left\{r: w \in \mathcal{A}_{r}\right\}$ we get that $w \in \mathcal{A}_{\frac{p}{\eta}}$. It follows that $M: L^{p / \eta}\left(\ell_{q / \eta}, w\right) \rightarrow L^{p / \eta}\left(\ell_{q / \eta}, w\right)$ is continuous. We thus obtain

$$
\|f\|_{R F_{p, q}^{s}(w)} \leq C\left\|\left(\sum_{\mu \geq 0}\left(\sum_{k \geq 1}\left(2^{\mu s}\left|\lambda_{\mu, k}\right| \chi_{\mu, k}\right)^{\eta}\right)^{\frac{q}{\eta}}\right)^{\frac{\eta}{q}}\right\|_{L_{\frac{p}{\eta}}\left(\mathbb{R}^{n}, w\right)}^{\frac{1}{\eta}} .
$$

Since for given $\mu$ the annuli $A_{\mu, k}, k \geq 1$, are essentially disjoint we obtain

$$
\|f\|_{R F_{p, q}^{s}(w)} \leq C\left\|\left(\sum_{\mu \geq 0} \sum_{k \geq 1}\left(2^{\mu s}\left|\lambda_{\mu, k}\right| \chi_{\mu, k}\right)^{q}\right)^{\frac{1}{q}}\right\|_{L_{p}\left(\mathbb{R}^{n}, w\right)}=C\|\lambda\|_{f_{p, q}^{s}(w)} .
$$

The analogous statement for weighted Besov spaces reads as follows:

Theorem 3.2. Let $p, q \in[1, \infty]$ and $w \in \mathcal{A}_{\infty}$. Then, the operators

$$
S: f \in R B_{p, q}^{s}\left(\mathbb{R}^{n}, w\right) \rightarrow\left(\left\langle f, \varphi_{\mu, k}\right\rangle\right)_{\mu, k} \in b_{p, q}^{s}(w)
$$

and

$$
T: \lambda \in b_{p, q}^{s}(w) \rightarrow \sum_{\mu \geq 0} \sum_{k \geq 1} \lambda_{\mu, k} \psi_{\mu, k} \in R B_{p, q}^{s}\left(\mathbb{R}^{n}, w\right)
$$

are bounded, and the composition $T \circ S$ is the identity on $R B_{p, q}^{s}\left(\mathbb{R}^{n}, w\right)$. In particular, $\|f\|_{R B_{p, q}^{s}(w)} \simeq\|S(f)\|_{b_{p, q}^{s}(w)}$. The same result holds also for the homogeneous version of these spaces. 
Proof. The unweighted case $w=1$ corresponds to [9, Theorems 5.1 and 5.2].

For the continuity of $S$, as in the proof of the previous theorem, we obtain that

$$
\sum_{k \geq 1} 2^{\mu s}\left|\left(\left\langle f, \varphi_{\mu, k}\right\rangle\right)\right| \chi_{\mu, k}(x) \leq C 2^{\mu s}\left|\varphi_{\mu}^{*} f(x)\right| \text { a.e. }
$$

where $\varphi_{\mu}^{*}$ is the Peetre maximal function for a given $\lambda>0$. Taking $\lambda$ big enough and using Theorem 2.2 we have

$$
\|S(f)\|_{b_{p, q}^{s}(w)} \leq C\left(\sum_{\mu \geq 0} 2^{\mu s q}\left\|\varphi_{\mu}^{*} f\right\|_{L^{p}\left(\mathbb{R}^{n}, w\right)}^{q}\right)^{\frac{1}{q}} \leq C\|f\|_{R B_{p, q}^{s}(w)} .
$$

For the continuity of $T$, fix $\lambda \in b_{p, q}^{s}(w)$ and let $f=\sum_{\mu \geq 0} \sum_{k \geq 1} \lambda_{\mu, k} \psi_{\mu, k}$. Then, arguing similarly as in the Triebel-Lizorkin case we see that for any $\mu \geq 0$,

$$
\begin{aligned}
\left\|\varphi_{\mu} * f\right\|_{L^{p}\left(\mathbb{R}^{n}, w\right)} & \leq C \sum_{\nu=\mu-1}^{\mu+1}\left\|\left(M\left(\sum_{k \geq 1}\left|\lambda_{\nu, k}\right|^{\eta} \chi_{\nu, k}^{\eta}\right)\right)^{\frac{1}{\eta}}\right\|_{L^{p}\left(\mathbb{R}^{n}, w\right)} \\
& =C \sum_{\nu=\mu-1}^{\mu+1}\left\|M\left(\sum_{k \geq 1}\left|\lambda_{\nu, k}\right|^{\eta} \chi_{\nu, k}^{\eta}\right)\right\|_{L^{p / \eta}\left(\mathbb{R}^{n}, w\right)}^{1 / \eta} \cdot
\end{aligned}
$$

Since $w \in \mathcal{A}_{\infty}$, setting as before $r_{0}:=\inf \left\{r: w \in \mathcal{A}_{r}\right\}$ and taking $\eta$ small enough to have $r_{0}<p / \eta$ we get that $w \in \mathcal{A}_{\frac{p}{\eta}}$ so that the maximal operator $M: L^{p / \eta}\left(\mathbb{R}^{n}, w\right) \rightarrow$ $L^{p / \eta}\left(\mathbb{R}^{n}, w\right)$ is continous. Then

$$
\begin{aligned}
\left\|M\left(\sum_{k \geq 1}\left|\lambda_{\nu, k}\right|^{\eta} \chi_{\nu, k}^{\eta}\right)\right\|_{L^{p / \eta}\left(\mathbb{R}^{n}, w\right)}^{1 / \eta} & \leq C\left\|\sum_{k \geq 1}\left|\lambda_{\nu, k}\right|{ }^{\eta} \chi_{\nu, k}^{\eta}\right\|_{L^{p / \eta}\left(\mathbb{R}^{n}, w\right)}^{1 / \eta} \\
& =C\left\|\sum_{k \geq 1}\left|\lambda_{\nu, k}\right| \chi_{\nu, k}\right\|_{L^{p}\left(\mathbb{R}^{n}, w\right)},
\end{aligned}
$$

where we have used the fact that for given $\nu$, the annuli $A_{\nu, k}$ are essentially disjoint. We deduce that

$$
\begin{aligned}
\|f\|_{R B_{p, q}^{s}(w)}^{q} & =\sum_{\mu \geq 0} 2^{\mu s q}\left\|\varphi_{\mu} * f\right\|_{L^{p}\left(\mathbb{R}^{n}, w\right)}^{q} \\
& \leq C \sum_{\mu \geq 0} 2^{\mu s q} \sum_{\nu=\mu-1}^{\mu+1}\left\|\sum_{k \geq 1}\left|\lambda_{\nu, k}\right| \chi_{\nu, k}\right\|_{L^{p}\left(\mathbb{R}^{n}, w\right)}^{q} \\
& \leq C \sum_{\mu \geq 0} 2^{\mu s q}\left\|\sum_{k \geq 1}\left|\lambda_{\nu, k}\right| \chi_{\nu, k}\right\|_{L^{p}\left(\mathbb{R}^{n}, w\right)}^{q} \\
& =C\|\lambda\|_{b_{p, q}^{s}(w)}^{q}
\end{aligned}
$$

\section{Continous and compact embeddings of Weighted Radial Besov SPACES}

In this section we use Theorem 3.2 to obtain sufficient conditions for the continuity and compactness of the embeddings of weighted radial Besov spaces, and apply these results to some relevant examples. 
Theorem 4.1. Let $p_{1}, p_{2}, q_{1}, q_{2} \in[1, \infty]$ and $w_{1}, w_{2}$ be $\mathcal{A}_{\infty}$-weights. There is a continuous embedding $R B_{p_{1}, q_{1}}^{s_{1}}\left(\mathbb{R}^{n}, w_{1}\right) \rightarrow R B_{p_{2}, q_{2}}^{s_{2}}\left(\mathbb{R}^{n}, w_{2}\right)$ provided that

$$
\left\{2^{-\mu\left(s_{1}-s_{2}\right)}\left\|\left\{\frac{w_{\mu k}^{2}}{w_{\mu k}^{1}}\right\}_{k}\right\|_{\ell_{p *}}\right\}_{\mu} \in \ell_{q *}
$$

where

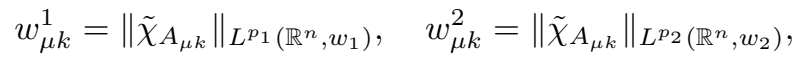

and

$$
\frac{1}{p^{*}}:=\left(\frac{1}{p_{2}}-\frac{1}{p_{1}}\right)_{+}, \quad \frac{1}{q^{*}}:=\left(\frac{1}{q_{2}}-\frac{1}{q_{1}}\right)_{+} .
$$

The embedding is compact provided that (4.1) holds and moreover

$$
\begin{aligned}
& \lim _{\mu \rightarrow+\infty} 2^{\mu\left(s_{2}-s_{1}\right)}\left\|\left\{\frac{w_{\mu k}^{2}}{w_{\mu k}^{1}}\right\}_{k}\right\|_{\ell_{p *}}=0 \quad \text { if } q^{*}=\infty \\
& \lim _{|k| \rightarrow+\infty} \frac{w_{\mu k}^{1}}{w_{\mu k}^{2}}=\infty \quad \text { for all } \mu \geq 0 \text { if } p^{*}=\infty .
\end{aligned}
$$

Proof. By Theorem 3.2 it suffices to study the embedding of the corresponding sequence spaces

$$
b_{p_{1}, q_{1}}^{s_{1}}\left(w_{1}\right) \rightarrow b_{p_{2}, q_{2}}^{s_{2}}\left(w_{2}\right)
$$

that is, using the notation of $[17$, section 3$]$,

$$
\ell_{q_{1}}\left(2^{\mu s_{1}} \ell_{p_{1}}\left(w_{1}\right)\right) \rightarrow \ell_{q_{2}}\left(2^{\mu s_{2}} \ell_{p_{2}}\left(w_{2}\right)\right)
$$

Notice that the continuity of this embedding is equivalent to the continuity of the embedding

$$
\ell_{q_{1}}\left(2^{\mu\left(s_{1}-s_{2}\right)} \ell_{p_{1}}\left(\frac{w_{1}}{w_{2}}\right)\right) \rightarrow \ell_{q_{2}}\left(\ell_{p_{2}}\right)
$$

Indeed

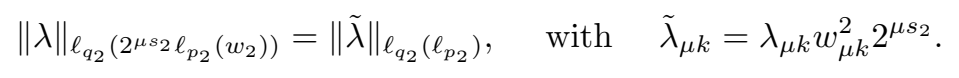

We can rewrite this embedding using the notation of [17] as

$$
\ell_{q_{1}}\left(\beta_{\mu} \ell_{p_{1}}(w)\right) \rightarrow \ell_{q_{2}}\left(\ell_{p_{2}}\right) \quad \text { with } \quad \beta_{\mu}=2^{\mu\left(s_{1}-s_{2}\right)}, w=\left(w_{\mu k}\right)_{\mu k}, w_{\mu k}=\frac{w_{\mu k}^{1}}{w_{\mu k}^{2}} .
$$

According to [17, Theorem 3.1], this embedding is continuous if and only if

$$
\left(\beta_{\mu}^{-1}\left\|\left(w_{\mu k}^{-1}\right)_{k}\right\|_{\ell_{p^{*}}}\right)_{\mu} \in \ell_{q_{*}}
$$

which proves that $R B_{p_{1}, q_{1}}^{s_{1}}\left(w_{1}\right) \subseteq R B_{p_{2}, q_{2}}^{s_{2}}\left(w_{2}\right)$ if (4.1) holds.

This embedding is compact if moreover

$$
\begin{aligned}
& \lim _{\mu \rightarrow+\infty} \beta_{\mu}^{-1}\left\|\left(w_{\mu k}^{-1}\right)_{k}\right\|_{\ell_{p^{*}}}=0 \quad \text { if } q^{*}=\infty \\
& \lim _{|k| \rightarrow+\infty} w_{\mu k}=\infty \quad \text { for all } \mu \geq 0 \text { if } p^{*}=\infty .
\end{aligned}
$$

which proves the theorem. 
As an example of application we now consider the case $w_{1}(x)=|x|^{\gamma_{1}}, w_{2}(x)=$ $|x|^{\gamma_{2}}$ with $\gamma_{1}, \gamma_{2}>-n$ so that $w_{1}, w_{2}$ are $\mathcal{A}_{\infty}$-weights. In order to simplify the statement of the following examples we introduce

$$
\delta:=s_{1}-\frac{n}{p_{1}}-s_{2}+\frac{n}{p_{2}} .
$$

Example 4.1. Let $p_{1}, p_{2}, q_{1}, q_{2} \in[1, \infty]$ and $\gamma_{1}, \gamma_{2}>-n$. There is a continuous embedding $R B_{p_{1}, q_{1}}^{s_{1}}\left(\mathbb{R}^{n},|x|^{\gamma_{1}}\right) \rightarrow R B_{p_{2}, q_{2}}^{s_{2}}\left(\mathbb{R}^{n},|x|^{\gamma_{2}}\right)$ provided that

$$
\left\{\begin{array} { l l } 
{ \frac { \gamma _ { 1 } } { p _ { 1 } } - \frac { \gamma _ { 2 } } { p _ { 2 } } \geq ( n - 1 ) ( \frac { 1 } { p _ { 2 } } - \frac { 1 } { p _ { 1 } } ) } & { \text { if } p ^ { * } = \infty } \\
{ \frac { \gamma _ { 1 } } { p _ { 1 } } - \frac { \gamma _ { 2 } } { p _ { 2 } } > \frac { n } { p * } } & { \text { if } p ^ { * } < \infty }
\end{array} \quad \text { and } \quad \left\{\begin{array}{ll}
\delta \geq \frac{\gamma_{1}}{p_{1}}-\frac{\gamma_{2}}{p_{2}} & \text { if } q^{*}=\infty \\
\delta>\frac{\gamma_{1}}{p_{1}}-\frac{\gamma_{2}}{p_{2}} & \text { if } q^{*}<\infty
\end{array}\right.\right.
$$

where $\delta$ is as in (4.2). This embedding is compact provided that the previous conditions hold and moreover

$$
\frac{\gamma_{1}}{p_{1}}-\frac{\gamma_{2}}{p_{2}}>(n-1)\left(\frac{1}{p_{2}}-\frac{1}{p_{1}}\right) \quad \text { if } p^{*}=\infty \quad \text { and } \quad \delta>\frac{\gamma_{1}}{p_{1}}-\frac{\gamma_{2}}{p_{2}} \quad \text { if } q^{*}=\infty .
$$

Proof. Since $|x| \sim k 2^{-\mu}$ for $x \in A_{\mu k}$, we have for $i=1,2$ that

$$
w_{\mu k}^{i}=\left\|\tilde{\chi}_{A_{\mu k}}\right\|_{L^{p_{i}}\left(|x|^{\gamma_{i}}\right)} \sim\left|A_{\mu k}\right|^{-1 / 2}\left(\left(k 2^{-\mu}\right)^{\gamma_{i}}\left|A_{\mu k}\right|\right)^{\frac{1}{p_{i}}} .
$$

Moreover $\left|A_{\mu k}\right| \sim k^{n-1} 2^{-\mu n}$. Hence

$$
\frac{w_{\mu k}^{2}}{w_{\mu k}^{1}} \sim 2^{\mu\left(\frac{n+\gamma_{1}}{p_{1}}-\frac{n+\gamma_{2}}{p_{2}}\right)} k^{\frac{\gamma_{2}}{p_{2}}-\frac{\gamma_{1}}{p_{1}}+(n-1)\left(\frac{1}{p_{2}}-\frac{1}{p_{1}}\right)} .
$$

Then if e.g. $p^{*}, q^{*}<\infty$ then (4.1) writes

$$
\sum_{k} k^{p^{*}\left(\frac{\gamma_{2}}{p_{2}}-\frac{\gamma_{1}}{p_{1}}+(n-1)\left(\frac{1}{p_{2}}-\frac{1}{p_{1}}\right)\right)}<\infty \quad \text { and } \quad \sum_{\mu} 2^{\mu q^{*}\left(\frac{\gamma_{1}}{p_{1}}-\frac{\gamma_{2}}{p_{2}}-\delta\right)}<\infty
$$

i.e.

$$
p^{*}\left(\frac{\gamma_{2}}{p_{2}}-\frac{\gamma_{1}}{p_{1}}+(n-1)\left(\frac{1}{p_{2}}-\frac{1}{p_{1}}\right)\right)<-1 \quad \text { and } \quad q^{*}\left(\frac{\gamma_{1}}{p_{1}}-\frac{\gamma_{2}}{p_{2}}-\delta\right)<0 .
$$

Recalling the definition of $p^{*}, q^{*}$ this gives the statement.

Concerning the compactness we have

$$
2^{\mu\left(s_{2}-s_{1}\right)}\left\{\sum_{k}\left(\frac{w_{\mu k}^{2}}{w_{\mu k}^{1}}\right)^{p^{*}}\right\}^{\frac{1}{p^{*}}} \sim 2^{\mu\left(\frac{\gamma_{1}}{p_{1}}-\frac{\gamma_{2}}{p_{2}}-\delta\right)}\left\{\sum_{k} k^{p^{*}\left(\frac{\gamma_{2}}{p_{2}}-\frac{\gamma_{1}}{p_{1}}+(n-1)\left(\frac{1}{p_{2}}-\frac{1}{p_{1}}\right)\right)}\right\}^{\frac{1}{p^{*}}}
$$

where the sum in the right hand side is finite.

Remark 4.1. (1) It is immediate form the above example that one has an improvement with respect to the non-radial case, c.f. [12, Proposition 2.8]. Indeed, in the case $p^{*}=\infty$ (that is, $p_{1} \leq p_{2}$ ) we can have $\frac{\gamma_{1}}{p_{1}}-\frac{\gamma_{2}}{p_{2}}<0$, in which case $\delta$ can be negative as well, while in the non-radial case both values must be non-negative.

(2) An alternative proof of the continuity part of the above example can be found in [7, Theorem 12]. For the corresponding non-radial case see [19, Theorem $1.1]$. 
Our next examples concern weights of purely polynomial growth. To this end, let $w_{\alpha, \beta}=\left\{\begin{array}{ll}|x|^{\alpha} & \text { if }|x| \leq 1 \\ |x|^{\beta} & \text { if }|x|>1\end{array}\right.$ with $\alpha, \beta>-n$.

Example 4.2. Let $-\infty<s_{2} \leq s_{1}<\infty, 0<p_{1}<\infty, 0<p_{2} \leq \infty$ and $0<q_{1}, q_{2} \leq$ $\infty$. Then, there is a continuous embedding $R B_{p_{1}, q_{1}}^{s_{1}}\left(\mathbb{R}^{n}, w_{\alpha, \beta}\right) \rightarrow R B_{p_{2}, q_{2}}^{s_{2}}\left(\mathbb{R}^{n}\right)$ provided

$$
\begin{cases}\frac{\beta}{p_{1}} \geq(n-1)\left(\frac{1}{p_{2}}-\frac{1}{p_{1}}\right) & \text { if } p^{*}=\infty \\ \frac{\beta}{p_{1}}>\frac{n}{p^{*}} & \text { if } p^{*}<\infty\end{cases}
$$

and one of the following conditions is satisfied:

$$
\begin{cases}\delta \geq \max \left(\frac{\alpha}{p_{1}},(n-1)\left(\frac{1}{p_{2}}-\frac{1}{p_{1}}\right)\right) & \text { if } q^{*}=\infty, p^{*}=\infty \\ \delta>\max \left(\frac{\alpha}{p_{1}},(n-1)\left(\frac{1}{p_{2}}-\frac{1}{p_{1}}\right)\right) & \text { if } q^{*}<\infty, p^{*}=\infty \\ \delta \geq \max \left(\frac{\alpha}{p_{1}}, \frac{n}{p^{*}}\right) & \text { if } q^{*}=\infty, p^{*}<\infty, \frac{n}{p^{*}} \neq \frac{\alpha}{p_{1}} \\ \delta>\max \left(\frac{\alpha}{p_{1}}, \frac{n}{p^{*}}\right) & \text { otherwise }\end{cases}
$$

Moreover the embedding $R B_{p_{1}, q_{1}}^{s_{1}}\left(\mathbb{R}^{n}, w_{\alpha, \beta}\right) \rightarrow R B_{p_{2}, q_{2}}^{s_{2}}\left(\mathbb{R}^{n}\right)$ is compact provided that

$$
\begin{cases}\frac{\beta}{p_{1}}>(n-1)\left(\frac{1}{p_{2}}-\frac{1}{p_{1}}\right) & \text { if } p^{*}=\infty \\ \frac{\beta}{p_{1}}>\frac{n}{p^{*}} & \text { if } p^{*}<\infty\end{cases}
$$

and

$$
\begin{cases}\delta>\max \left(\frac{\alpha}{p_{1}},(n-1)\left(\frac{1}{p_{2}}-\frac{1}{p_{1}}\right)\right) & \text { if } p^{*}=\infty \\ \delta>\max \left(\frac{\alpha}{p_{1}}, \frac{n}{p^{*}}\right) & \text { if } p^{*}<\infty\end{cases}
$$

Proof. Consider first the Besov case. We have

$$
\frac{w_{\mu k}^{2}}{w_{\mu k}^{1}} \sim k^{(n-1)\left(\frac{1}{p_{2}}-\frac{1}{p_{1}}\right)} 2^{-\mu n\left(\frac{1}{p_{2}}-\frac{1}{p_{1}}\right)} \times \begin{cases}k^{-\frac{\alpha}{p_{1}}} 2^{\frac{\mu \alpha}{p_{1}}} & \text { if } k \leq 2^{\mu} \\ k^{-\frac{\beta}{p_{1}}} 2^{\frac{\mu \beta}{p_{1}}} & \text { if } k>2^{\mu}\end{cases}
$$

Then if e.g. $p^{*}=\infty, q^{*}<\infty,(4.1)$ writes

$$
\sum_{\mu} 2^{\mu q^{*}\left[\left(s_{2}-s_{1}\right)-n\left(\frac{1}{p_{2}}-\frac{1}{p_{1}}\right)+\frac{\alpha}{p_{1}}\right]}\left(\sup _{k \leq 2^{\mu}} k^{(n-1)\left(\frac{1}{p_{2}}-\frac{1}{p_{1}}\right)-\frac{\alpha}{p_{1}}}\right)^{q^{*}}<\infty
$$

and

$$
\sum_{\mu} 2^{\mu q^{*}\left[\left(s_{2}-s_{1}\right)-n\left(\frac{1}{p_{2}}-\frac{1}{p_{1}}\right)+\frac{\beta}{p_{1}}\right]}\left(\sup _{k>2^{\mu}} k^{(n-1)\left(\frac{1}{p_{2}}-\frac{1}{p_{1}}\right)-\frac{\beta}{p_{1}}}\right)^{q^{*}}<\infty
$$

which gives the statement. As for the compactness, we need that

$$
\lim _{|k| \rightarrow \infty} k^{(n-1)\left(\frac{1}{p_{2}}-\frac{1}{p_{1}}\right)-\frac{\beta}{p_{1}}}=0 .
$$

The remaining cases are analogous.

The generalization to the following two-weighted embeddings is straightforward: 
Example 4.3. Let $-\infty<s_{2} \leq s_{1}<\infty, 0<p_{1}<\infty, 0<p_{2} \leq \infty$ and $0<q_{1}, q_{2} \leq \infty$. Then, there is a continuous embedding $R B_{p_{1}, q_{1}}^{s_{1}}\left(\mathbb{R}^{n}, w_{\alpha_{1}, \beta_{1}}\right) \rightarrow$ $R B_{p_{2}, q_{2}}^{s_{2}}\left(\mathbb{R}^{n}, w_{\alpha_{2}, \beta_{2}}\right)$ provided

$$
\begin{cases}\frac{\beta_{1}}{p_{1}}-\frac{\beta_{2}}{p_{2}} \geq(n-1)\left(\frac{1}{p_{2}}-\frac{1}{p_{1}}\right) & \text { if } p^{*}=\infty \\ \frac{\beta_{1}}{p_{1}}-\frac{\beta_{2}}{p_{2}}>\frac{n}{p^{*}} & \text { if } p^{*}<\infty\end{cases}
$$

and one of the following conditions is satisfied:

$$
\begin{cases}\delta \geq \max \left(\frac{\alpha_{1}}{p_{1}}-\frac{\alpha_{2}}{p_{2}},(n-1)\left(\frac{1}{p_{2}}-\frac{1}{p_{1}}\right)\right) & \text { if } q^{*}=\infty, p^{*}=\infty \\ \delta>\max \left(\frac{\alpha_{1}}{p_{1}}-\frac{\alpha_{2}}{p_{2}},(n-1)\left(\frac{1}{p_{2}}-\frac{1}{p_{1}}\right)\right) & \text { if } q^{*}<\infty, p^{*}=\infty \\ \delta \geq \max \left(\frac{\alpha_{1}}{p_{1}}-\frac{\alpha_{2}}{p_{2}}, \frac{n}{p^{*}}\right) & \text { if } q^{*}=\infty, p^{*}<\infty, \frac{n}{p^{*}} \neq \frac{\alpha_{1}}{p_{1}}-\frac{\alpha_{2}}{p_{2}} \\ \delta>\max \left(\frac{\alpha_{1}}{p_{1}}-\frac{\alpha_{2}}{p_{2}}, \frac{n}{p^{*}}\right) & \text { otherwise }\end{cases}
$$

where $\delta$ is as in (4.2).

Moreover the embedding $R B_{p_{1}, q_{1}}^{s_{1}}\left(\mathbb{R}^{n}, w_{\alpha_{1}, \beta_{1}}\right) \rightarrow R B_{p_{2}, q_{2}}^{s_{2}}\left(\mathbb{R}^{n}, w_{\alpha_{2}, \beta_{2}}\right), p_{1}, p_{2} \in$ $(0, \infty)$, is compact provided that

$$
\begin{cases}\frac{\beta_{1}}{p_{1}}-\frac{\beta_{2}}{p_{2}}>(n-1)\left(\frac{1}{p_{2}}-\frac{1}{p_{1}}\right) & \text { if } p^{*}=\infty \\ \frac{\beta_{1}}{p_{1}}-\frac{\beta_{2}}{p_{2}}>\frac{n}{p^{*}} & \text { if } p^{*}<\infty\end{cases}
$$

and one of the following conditions is satisfied:

$$
\begin{cases}\delta>\max \left(\frac{\alpha_{1}}{p_{1}}-\frac{\alpha_{2}}{p_{2}},(n-1)\left(\frac{1}{p_{2}}-\frac{1}{p_{1}}\right)\right) & \text { if } p^{*}=\infty \\ \delta>\max \left(\frac{\alpha_{1}}{p_{1}}-\frac{\alpha_{2}}{p_{2}}, \frac{n}{p^{*}}\right) & \text { if } p^{*}<\infty\end{cases}
$$

\section{Continuous and COMPaCt Embeddings of Weighted Radial TRIEBEL-LIZORKIN SPACES}

Our next result concers embeddings for Triebel-Lizorkin spaces with radial $\mathcal{A}_{\infty}$ weights. We will follow the approach in [20], which is based on a GagliardoNirenberg type inequality and two lemmas on products of Muckenhoupt weights that we recall for the reader's convenience.

Proposition 5.1. [19, Proposition 5.1] Let $q, q_{0}, q_{1} \in[1, \infty]$ and $\theta \in(0,1)$. Let $p, p_{0}, p_{1} \in(1, \infty)$ and $-\infty<s_{0}<s_{1}<\infty$ satisfy

$$
\frac{1}{p}=\frac{1-\theta}{p_{0}}+\frac{\theta}{p_{1}} \text { and } s=(1-\theta) s_{0}+\theta s_{1} .
$$

Let further $w, w_{0}, w_{1} \in \mathcal{A}_{\infty}$ be such that $w=w_{0}^{(1-\theta) p / p_{0}} w_{1}^{\theta p / p_{1}}$. Then there exists a constant $C$ such that for all $f \in \mathcal{S}^{\prime}\left(\mathbb{R}^{n}\right)$ one has

$$
\|f\|_{F_{p, q}^{s}(w)} \leq C\|f\|_{F_{p_{0}, q_{0}}^{s_{0}}\left(w_{0}\right)}^{1-\theta}\|f\|_{F_{p_{1}, q_{1}}^{s_{1}}\left(w_{1}\right)}^{\theta} .
$$

Lemma 5.1. [20, Lemma 3.1] Let $1<p<\infty$ and $w_{1}, w_{2} \in \mathcal{A}_{p}$. Then, there is $\eta_{0}>0$ such that, for all $\varepsilon, \delta \in\left[0, \eta_{0}\right)$ one has $w_{1}^{-\varepsilon} w_{2}^{1+\delta} \in \mathcal{A}_{p}$.

Lemma 5.2. [20, Lemma 3.2] Let $w_{1}, w_{2} \in \mathcal{A}_{\infty}$. Then there are $\eta_{0}>0$ and a constant $C>0$ such that for all $\varepsilon, \delta \in\left(0, \eta_{0}\right)$ and all cubes $Q \subset \mathbb{R}^{n}$ we have

$$
\int_{Q} w_{1}^{-\varepsilon} w_{2}^{1+\delta} d x \leq C|Q|^{\varepsilon-\delta}\left(\int_{Q} w_{1} d x\right)^{-\varepsilon}\left(\int_{Q} w_{2} d x\right)^{1+\delta} .
$$


Since our functions are supported on annuli instead of cubes, we will need another auxiliary lemma on the behavior of products of radial Muckenhoupt weights over these sets. To this end, we first recall the following characterization of radial $\mathcal{A}_{p}$ weights given by Duoandikoetxea et al. in [8]:

Lemma 5.3. [8, Theorem 3.2] Let $w_{0}:(0, \infty) \rightarrow[0, \infty]$ and $w_{n}(x)=w_{0}(|x|)$ for $x \in \mathbb{R}^{n}$. Then $w_{n}$ is in $\mathcal{A}_{p}\left(\mathbb{R}^{n}\right)$ if and only if $\delta_{n} w_{0}$ is in $\mathcal{A}_{p}(0,+\infty)$, where $\delta_{n} w_{0}(t)=w_{0}\left(t^{1 / n}\right)$.

Lemma 5.4. Let $w_{1}, w_{2} \in \mathcal{A}_{\infty}, w_{1}(x)=\tilde{w}_{1}(|x|), w_{2}(x)=\tilde{w}_{2}(|x|)$ for all $x \in \mathbb{R}^{n}$. Then, there exists $\eta_{0}>0$ such that for all $\varepsilon \in\left(0, \eta_{0}\right)$ and any annnulus $D_{a b}=\{x \in$ $\left.\mathbb{R}^{n}: a \leq|x| \leq b\right\}, a, b \in \mathbb{R}_{+}$,

$$
\int_{D_{a b}} w_{1}^{-\varepsilon} w_{2}^{1+\varepsilon} d x \leq C\left(\int_{D_{a b}} w_{1} d x\right)^{-\varepsilon}\left(\int_{D_{a b}} w_{2} d x\right)^{1+\varepsilon}
$$

Proof. Fix $p>1$ such that $w_{1}, w_{2} \in \mathcal{A}_{p}$, let $\eta_{0}$ be as in Lemma 5.2 and $\varepsilon \in\left(0, \eta_{0}\right)$. Taking polar coordinates we obtain

$$
\begin{aligned}
\int_{D_{a b}} w_{1}^{-\varepsilon} w_{2}^{1+\varepsilon} d x & =\omega_{n-1} \int_{a}^{b} \tilde{w}_{1}^{-\varepsilon} \tilde{w}_{2}^{1+\varepsilon} r^{n-1} d r \\
& =\omega_{n-1} \int_{a^{n}}^{b^{n}}\left(\delta_{n} \tilde{w}_{1}\right)^{-\varepsilon}\left(\delta_{n} \tilde{w}_{2}\right)^{1+\varepsilon} \frac{d r}{n} \\
& \leq C \omega_{n-1}\left(\int_{a}^{b} \delta_{n} \tilde{w}_{1} d r\right)^{-\varepsilon}\left(\int_{a}^{b} \delta_{n} \tilde{w}_{2} d r\right)^{1+\varepsilon}
\end{aligned}
$$

where the last bound follows from Lemma 5.2, and we have used the fact that $\delta_{n} \tilde{w}_{1}, \delta_{n} \tilde{w}_{2} \in \mathcal{A}_{p}(0,+\infty)$ by Lemma 5.3. Changing variables again we obtain the desired bound.

Now we are ready to prove our result for Triebel-Lizorkin spaces:

Theorem 5.1. Let $1 \leq p_{1} \leq p_{2} \leq \infty, q_{1}, q_{2} \in[1, \infty]$ and $w_{1}, w_{2}$ be radially symmetric $\mathcal{A}_{\infty}$-weights. There is a continuous embedding $R F_{p_{1}, q_{1}}^{s_{1}}\left(\mathbb{R}^{n}, w_{1}\right) \rightarrow$ $R F_{p_{2}, q_{2}}^{s_{2}}\left(\mathbb{R}^{n}, w_{2}\right)$ provided that

$$
\sup _{\mu, k}\left\{2^{-\mu\left(s_{1}-s_{2}\right)} \frac{w_{\mu k}^{2}}{w_{\mu k}^{1}}\right\}<+\infty
$$

where

$$
w_{\mu k}^{1}=\left\|\tilde{\chi}_{A_{\mu k}}\right\|_{L^{p_{1}}\left(\mathbb{R}^{n}, w_{1}\right)}, \quad w_{\mu k}^{2}=\left\|\tilde{\chi}_{A_{\mu k}}\right\|_{L^{p_{2}\left(\mathbb{R}^{n}, w_{2}\right)},}
$$

The embedding is compact provided that (5.1) holds and

$$
\lim _{|k| \rightarrow+\infty} \frac{w_{\mu k}^{1}}{w_{\mu k}^{2}}=\infty \quad \text { for all } \mu \geq 0 .
$$

Proof. The proof has two steps: proving the continuity of the embedding and then the compactness.

For the first part, we follow closely the approach in [20], which we outline for the reader's convenience. Note that it suffices to prove the continuity of the embedding $R F_{p_{1}, q_{1}}^{s_{1}}\left(\mathbb{R}^{n}, w_{1}\right) \hookrightarrow R F_{p_{2}, q_{2}}^{s_{2}}\left(\mathbb{R}^{n}, w_{2}\right)$ with $q_{2} \leq \min \left\{p_{1}, p_{2}\right\}$ since then the result follows from Theorem 2.1(3). 
Since $p_{2} \geq p_{1}$, there exists $\theta_{0} \in[0,1)$ such that $\frac{1}{p_{2}}-\frac{1-\theta_{0}}{p_{1}}=0$ (in fact, $\theta_{0}=\frac{p_{2}}{p_{1}}-1$ ). For $\theta \in\left(\theta_{0}, 1\right)$, let

$$
\varepsilon=\frac{\frac{1-\theta}{p_{1}}}{\frac{1}{p_{2}}-\frac{1-\theta}{p_{1}}}>0
$$

which clearly tends to zero as $\theta \rightarrow 1$, and let $v, r, t$ be defined by the identities

$$
v=w_{1}^{-\varepsilon} w_{2}^{1+\varepsilon}, \quad \frac{1}{p_{2}}=\frac{1-\theta}{p_{1}}+\frac{\theta}{r}, \quad s_{2}=(1-\theta) s_{1}+\theta t .
$$

Then, one can check that $w_{2}=w_{1}^{(1-\theta) p_{2} / p_{1}} v^{p_{2} \theta / r}, r \in\left[p_{2},+\infty\right)$ and $t<s_{2}<s_{1}$. Moreover, $v \in A_{p_{2}} \subseteq A_{r}$ if $\theta$ is sufficiently close to 1 by Lemma 5.1 . Hence, by Proposition 5.1, it holds

$$
\|f\|_{R F_{p_{2}, q_{2}}^{s_{2}}\left(\mathbb{R}^{n}, w_{2}\right)} \leq C\|f\|_{R F_{p_{1}, q_{1}}^{s_{1}}\left(\mathbb{R}^{n}, w_{1}\right)}^{1-\theta_{1}}\|f\|_{R F_{r, r}^{t}\left(\mathbb{R}^{n}, v\right)}^{\theta}
$$

Now, since $R B_{p, p}^{s}=R F_{p, p}^{s}$ and $r \geq p_{2}$, by Theorem 4.1

$$
\|f\|_{R F_{r, r}^{t}\left(\mathbb{R}^{n}, v\right)} \leq C\|f\|_{R F_{p_{2}, p_{2}}^{s_{2}}\left(\mathbb{R}^{n}, w_{2}\right)}
$$

holds provided that

$$
\sup _{k, \mu} 2^{-\mu\left(s_{2}-t\right)}\left(\int_{A_{\mu k}} v\right)^{1 / r}\left(\int_{A_{\mu k}} w_{2}\right)^{1 / p_{2}}<+\infty .
$$

But, by Lemma 5.4

$$
\int_{A_{\mu k}} v \leq C\left(\int_{A_{\mu k}} w_{1}\right)^{-\varepsilon}\left(\int_{A_{\mu k}} w_{2}\right)^{1+\varepsilon}
$$

whence, replacing this bound into (5.3) and noting that $s_{2}-t=\left(s_{1}-s_{2}\right) \frac{1-\theta}{\theta}$, $\frac{\varepsilon}{r}=\frac{1}{p_{1}} \frac{1-\theta}{\theta}$, and $\frac{1+\varepsilon}{r}=\frac{1}{\theta p_{2}}$, the desired bound finally follows from condition (5.1).

Since $q_{2} \leq p_{2}$ by the above assumption, we may replace $R F_{p_{2}, p_{2}}^{s_{2}}$ on the right hand side of (5.2) by $R F_{p_{2}, q_{2}}^{s_{2}}$, and divide by $\|f\|_{R F_{p_{2}, q_{2}}^{s_{2}}\left(\mathbb{R}^{n}, w_{2}\right)}^{\theta}$ to obtain the bound

$$
\|f\|_{R F_{p_{2}, q_{2}}^{s_{2}}\left(\mathbb{R}^{n}, w_{2}\right)} \leq C\|f\|_{R F_{p_{1}, q_{1}}^{s_{1}}\left(\mathbb{R}^{n}, w_{1}\right)} .
$$

Notice that, in principle, this bound holds in the intersection $R F_{p_{1}, q_{1}}^{s_{1}}\left(\mathbb{R}^{n}, w_{1}\right) \cap$ $R F_{p_{2}, q_{2}}^{s_{2}}\left(\mathbb{R}^{n}, w_{2}\right)$, but it can be extended by density to $R F_{p_{1}, q_{1}}^{s_{1}}\left(\mathbb{R}^{n}, w_{1}\right.$ ) (see $[20$, Proof of Theorem 1.2]).

It remains to prove that the embedding is compact. To this end, let $\left(f_{k}\right)_{k \in \mathbb{N}}$ be

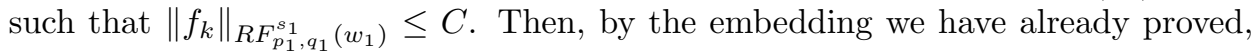
$\left(f_{k}\right)_{k \in \mathbb{N}}$ is also bounded in $R F_{p_{2}, q_{2}}^{s_{2}}\left(w_{2}\right)$ with $q_{2} \leq \min \left\{p_{1}, p_{2}\right\}$ and, by Theorem 2.1 in $R B_{p_{2}, p_{2}}^{s_{2}}\left(w_{2}\right)$. Since, under our hypotheses, the embedding $R B_{r, r}^{t}(v) \hookrightarrow$ $R B_{p_{2}, p_{2}}^{s_{2}}\left(w_{2}\right)$ is compact by Theorem 4.1 , we have that $f_{k} \rightarrow f$ in $B_{r, r}^{t}=F_{r, r}^{t}$. Then,

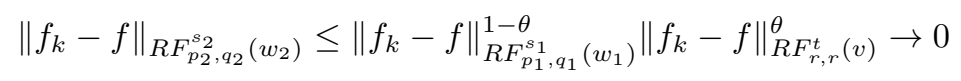

which proves our statement. 
Examples for the same weights considered in the Besov case can be obtained in an analogous manner. We leave the proofs to the reader.

An interesting special case of the inhomogenous Triebel-Lizorkin spaces is given by the Bessel potential spaces. In [6] the first two authors proved (with a more elementary argument) the following result.

Example 5.1. [6, Theorems 6.4 and 7.2] Let $1<p<\infty, 0<s<\frac{n}{p}, p \leq q \leq p_{c}^{*}=$ $\frac{p(n+c)}{n-s p}$. Then we have a continuous embedding

$$
H_{\text {rad }}^{s, p}\left(\mathbb{R}^{n}\right) \subset L^{q}\left(\mathbb{R}^{n},|x|^{c} d x\right)
$$

provided that

$$
-s p<c<\frac{(n-1)(q-p)}{p}
$$

Morevover, the embedding is compact when $p<q<p_{c}^{*}$.

Proof. To see this result as a special case of the embeddings in Theorem 5.1, notice that $H_{\text {rad }}^{s, p}=R F_{p, 2}^{s}$ and $L_{r a d}^{q}\left(|x|^{c}\right)=R F_{q, 2}^{0}\left(|x|^{c}\right)$ provided $|x|^{c} \in A_{q}$ (that is, $-n<$ $c<n(q-1))$. Hence, this case corresponds to the choice $w_{1}=1, w_{2}=|x| c, p_{1}=p$, $q_{1}=2, p_{2}=q$, and $q_{2}=2$. Moreover, since we are interested in the case $q \geq p$, this implies $p^{*}=\infty$, while $q^{*}=\infty$ by the choice of spaces. Therefore, we obtain $c<\frac{(n-1)(q-p)}{p}$ and $q<\frac{p(c+n)}{n-s p}$. The remaining conditions $c>-s p$ and $s<\frac{n}{p}$ are needed to have a non-empty interval of admissible values of $q$.

A different proof of the previous example for $p=2$ was also given in [5] by the first two authors jointly with R. Durán, where that result was used to analyze the existence of radial solutions of a weighted elliptic system with hamiltonian structure in $\mathbb{R}^{n}$.

\section{REFERENCES}

[1] K. F. Andersen and R. T. John. Weighted inequalities for vector-valued maximal functions and singular integrals, Studia Math. 69 (1980), 19-31.

[2] H.-Q. Bui, Weighted Besov and Triebel Lizorkin spaces: Interpolation by the real method. Hiroshima Math. J. 12, (1982), 581-605.

[3] H.-Q. Bui, M. Paluszyñski, M. R. Taibleson. A maximal characterization of weighted BesovLipschitz and Triebel-Lizorkin spaces. Studia Math. 119 (3), (1996), 219-246.

[4] L. Cui, L. Peng. Biorthogonal radial multiresolution in dimension three. J. Comput. Appl. Math. 224 (2009), no. 2, 581-591.

[5] P. L. De Nápoli, I. Drelichman, R. G. Durán. Radial solutions for Hamiltonian elliptic systems with weights. Adv. Nonlinear Stud. 9 (2009), no. 3, 579-593.

[6] P. L. De Nápoli, I. Drelichman. Elementary proofs of embedding theorems for potential spaces of radial functions. Preprint 2014, arXiv:1404.7468.

[7] P. L. De Nápoli, I. Drelichman. Weighted convolution inequalities for radial functions. Ann. Mat. Pura Appl. 194 (2015), no. 1, 167-181.

[8] J. Duoandikoetxea, A. Moyua, O. Oruetxebarria, E. Seijo. Radial $A_{p}$ weights with applications to the disc multiplier and the Bochner-Riesz operators. Indiana Univ. Math. J. 57 (2008), no. 3, $1261-1281$.

[9] J. Epperson, M. Frazier. An almost orthogonal radial wavelet expansion for radial distributions. J. Fourier Anal. Appl. 1 (1995), no. 3, 311-353.

[10] J. Franke. On the spaces $F_{p, q}^{s}$ of Triebel-Lizorkin type: Pointwise multipliers and spaces on domains. Math. Nachr. 125 (1986), 29-68.

[11] J. Garcia-Cuerva, J. L. Rubio de Francia. Weighted norm inequalities and related topics. North-Holland, Amsterdam, 1985. 
[12] D. Haroske, L. Skrzypczak. Entropy and approximation numbers of embeddings of function spaces with Muckenhoupt weights. I. Rev. Mat. Complut. 21 (2008), no. 1, 135-177.

[13] D. Haroske, L. Skrzypczak. Entropy and approximation numbers of embeddings of function spaces with Muckenhoupt weights, II. General weights. Ann. Acad. Sci. Fenn. Math. 36 (2011), no. $1,111-138$.

[14] B. Jawerth. Some observations on Besov and Lizorkin-Triebel spaces. Math. Scand. 40 (1977), 94-104.

[15] V. M. Kokilasvili, Maximal inequalities and multipliers in weighted Lizorkin-Triebel spaces, Soviet Math. Dokl. 19 (1978), 271-276.

[16] T. Kühn, H-G. Leopold, W. Sickel, L. Skrzypczak. Entropy numbers of Sobolev embeddings of radial Besov spaces. J. Approx. Theory 121 (2003), no. 2, 244-268.

[17] T. Kühn, H-G. Leopold, W. Sickel, L. Skrzypczak. Entropy numbers of embeddings of weighted Besov spaces. II. Proc. Edinb. Math. Soc. (2) 49 (2006), no. 2, 331-359.

[18] P. L. Lions. Symétrie e compacité dans les espaces de Sobolev, J. Funct. Anal. 49 (1982), 315-334.

[19] M. Meyries, M. Veraar. Sharp embedding results for spaces of smooth functions with power weights. Studia Math. 208 (2012), no. 3, 257-293.

[20] M. Meyries, M. Veraar. Characterization of a class of embeddings for function spaces with Muckenhoupt weights. Preprint 2014, arXiv:1409.2396.

[21] W. M. Ni. A nonlinear Dirichlet problem on the unit ball and its applications. Indiana Univ. Math. J. 31 (1982), 801-807.

[22] J. Peetre. New thoughts on Besov spaces. Duke University Mathematics Series, No. 1. Mathematics Department, Duke University, Durham, N.C., 1976.

[23] J. Peetre. On spaces of Triebel-Lizorkin type. Ark. Mat. 13 (1975), 123-130.

[24] H. Rauhut, M. Rösler. Radial multiresolution in dimension three. Constr. Approx. 22 (2005), no. 2, 193-218.

[25] V. S. Rychkov. Littlewood-Paley Theory and Function Spaces with A loc $_{p}$ Weights. Math. Nachr. 224 (2001), 145-180

[26] W. Sickel, L. Skrzypczak. Radial subspaces of Besov and Lizorkin-Triebel classes: Extended Strauss lemma and compactness of embeddings. J. Fourier Anal. Appl. 6 (2000), 639-662.

[27] W. Sickel, L. Skrzypczak. On the interplay of regularity and decay in case of radial functions

II. Homogeneous spaces. J. Fourier Anal. Appl. 18 (2012), no. 3, 548-582.

[28] W. Sickel, L. Skrzypczak, J. Vybiral. On the interplay of regularity and decay in case of radial functions I. Inhomogeneous spaces. Commun. Contemp. Math. 14 (2012), no. 1, 1250005, $60 \mathrm{pp}$.

[29] W. A. Strauss. Existence of Solitary Waves in Higher Dimensions. Comm. Math. Phys. 55 (1977), 149-162.

[30] H. Triebel. Theory of function spaces. Mathematik und ihre Anwendungen in Physik und Technik, 38. Akademische Verlagsgesellschaft Geest \& Portig K.-G., Leipzig, 1983.

[31] H. Triebel. Theory of function spaces. III. Monographs in Mathematics, 100. Birkhäuser Verlag, Basel, 2006.

imas (UBA-COniCET) and Departamento de Matemática, Facultad de Ciencias ExaCtas y Naturales, Universidad de Buenos Aires, Ciudad Universitaria, 1428 Buenos Aires, Argentina

E-mail address: pdenapo@dm.uba.ar

imas (UBA-COnicet) and Departamento de Matemática, Facultad de Ciencias Exactas y Naturales, Universidad de Buenos Aires, Ciudad Universitaria, 1428 Buenos Aires, Argentina

E-mail address: irene@drelichman.com

Instituto de Ciencias, Universidad Nacional de Gral. Sarmiento, J. M. Gutierrez 1150, Los Polvorines, 1613 Provincia de Buenos Aires, Argentina and Departamento de Matemática, Facultad de Ciencias Exactas y Naturales, Universidad de Buenos Aires, Ciudad Universitaria, 1428 Buenos Aires, Argentina

E-mail address: nsaintie@dm.uba.ar 